\title{
Timeliness of Chlamydia Laboratory and Provider Reports: A Modern Perspective
}

\author{
Patrick T. Lai ${ }^{* 1,3}$, Janae E. Johns², Uzay Kirbiyik ${ }^{2,3}$ and Brian E. Dixon², 3 \\ 'Indiana University, School of Informatics and Computing, Indianapolis, IN, USA; ${ }^{2}$ Indiana University, Richard M. Fairbanks School of \\ Public Health, Indianapolis, IN, USA; ${ }^{3}$ Regenstrief Institute, Center for Biomedical Informatics, Indianapolis, IN, USA
}

\section{Objective}

To analyze the time delay between a chlamydia positive test diagnosis and when a laboratory and/or a provider sends a report to a local public health department.

\section{Introduction}

Timeliness of reports sent by laboratories and providers is a continuous challenge for disease surveillance and management. Public health organizations often collect communicable disease reports with various degrees of timeliness raising the concern about the delay in patient information received (1). Timely reports are beneficial to accurately evaluate community health needs and investigate disease outbreaks (2). According to Indiana state law, chlamydia reports are required to be sent to public health within 3 days after a positive test result confirmation (3). Therefore, laboratories and providers must be accountable and comply with regulation to ensure accurate data quality of disease assessment.

\section{Methods}

A sample of 2,428 chlamydia laboratory and provider reports were collected during the period from May 2012 through July 2012 from a local health department serving the Indianapolis area. Due to absence of test confirmation dates, dates that a report is sent to public health, and other missing data, only 1,752 reports were included in this study. The time delay was calculated by determining the difference between when the initial report is sent to public health following positive confirmatory test by the laboratory. Reports were differentiated as either a laboratory report or a provider report coming directly from a clinician or a hospital setting. Statistical analyses and frequency tables were conducted using SAS 9.4.

\section{Results}

Table 1 displays the counts of chlamydia laboratory and provider reports according to the time delay in days, the percentage of reports sent to public health within 3 days, and the summary statistics for the two types of reports with a graphical representation shown in Figure 1. There is a clear lag between a lab test and when a provider report is sent to public health. Negative binomial regression result was highly significant with $\mathrm{p}<0.001$.

\section{Conclusions}

This study shows the importance of continued examination of timeliness of disease reporting from both laboratory and provider settings. Most lab reports are received electronically and comply with state law. However, reports from providers tend to be fax-based and received later than the 72 hours desired by health officials. With the greater adoption of electronic health records (EHR), it might be possible to further enhance disease surveillance through more timely provider-based reporting which could also reduce the volume of missing data from provider reports like those observed with electronic lab reporting (2). Future research should examine EHR capacity and clinical workflows to improve provider-based reporting processes.

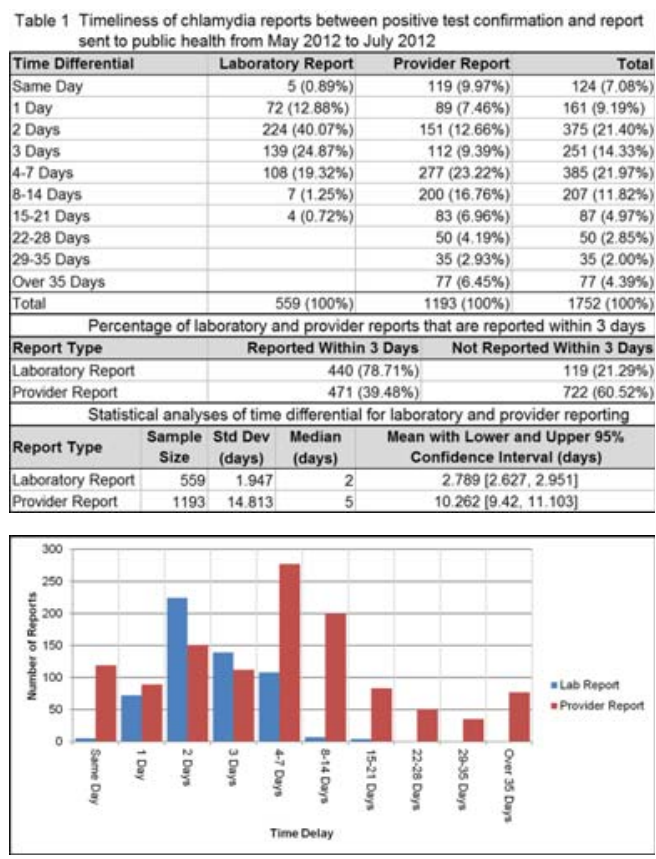

Figure 1 Frequency count and time delay of chlamydia reporting for laboratory and provider reports

\section{Keywords}

Public Health Reporting; Timeliness; Chlamydia; Data Quality; Communicable Disease Control

\section{Acknowledgments}

This project was supported by grant number R01HS020909 from the Agency for Healthcare Research and Quality. The content is solely the responsibility of the authors and does not necessarily represent the official views of AHRQ.

\section{References}

1) Overhage JM, Grannis S, McDonald CJ. A Comparison of the Completeness and Timeliness of Automated Electronic Laboratory Reporting and Spontaneous Reporting of Notifiable Conditions. American Journal of Public Health. 2008 Feb;98(2):344-50.

2) Dixon BE, McGowan JJ, Grannis SJ. Electronic Laboratory Data Quality and the Value of a Health Information Exchange to Support Public Health Reporting Processes. AMIA Annu Symp Proc. 2011;322-30.

3) Indiana Administrative Code: Title 410 Indiana State Department of Health. Indiana General Assembly. http://www.in.gov/legislative/iac/ iac title?iact $=410$.

\section{*Patrick T. Lai \\ E-mail: ptlai@imail.iu.edu}

